\title{
Smart KOT App Generation using an Android Application
}

\author{
M N Jayaram, Nisarga Gowda M A
}

\begin{abstract}
One of the maximum critical levels of serving your visitors is to take their orders correctly. It is taken into consideration to be the start line of the patron's evaluation of your services. It is likewise the maximum fundamental step within the system of serving the right food to the proper patron. It is incredible how era can revolutionize the best yet crucial practices of hoteliers, and the Kitchen Order Token / Ticket app is a tremendous example of the same. In the traditional method, the order is taken by the waiter manually by using a pen and paper that is referred to as the Kitchen Order Token / Ticket. Usually had to be printed as duplicates(or triplicates in a few cases), traditional Kitchen Order Token / Ticket techniques serve to create miscommunication, consume greater time, and are extra vulnerable to manual mistakes. In this paper mainly concentrate on the Android application is used in the android platform Tablets for taking orders from the customer near their tables only. The app is used by the waiters to take down the orders. The particular device will be given to the waiters in the organization with their username and password logged in respectively. This app will act as the user end application and the server will be the web application (server) that is managed by the Administrator.

Keywords: Android Device, Digital Menu, Laravel software, Mobile application, Mysql Server, PHP Language, Smartphone, Wireless food ordering system.
\end{abstract}

\section{INTRODUCTION}

In the existing world, it mainly depends on the internet and wireless technologies [2], there is more growth of online technologies in the area of hotel/restaurant filed like order-taking is been digitalized to enhance the performance of hotels[4]. Traditionally for food shops, customers want to do visit their favorite restaurants, during the peak hours the tables are not available they need to wait until there turn to get the table free.[3] When the waiter note down the order that is placed by a customer, waiter note the orders by using a traditional method using this it may misplace the order during the peak hours, to avoid these type of mistakes android tablets are been used to take the order through wirelessly, by using this kind of technology it reduces the service response time and serves food on time. Nowadays people use online technologies /wireless technologies more like online payments, net banking, etc, all this system is based on the wi-fi it provides the cloud storage. [9]Wi-Fi storage which can create the own cloud /private cloud is more secure than the public cloud and also handles the smooth file sharing, image sharing

Revised Manuscript Received on October 20, 2019.

* Correspondence Author

Dr M N Jayaram*, Assistant Professor, Department of Electronics and Communication, JSS Science and Technology University, Karnataka, India.

Nisarga Gowda M A, Department of Electronics and Communication, JSS Science and Technology University, Karnataka, India.

(C) The Authors. Published by Blue Eyes Intelligence Engineering and Sciences Publication (BEIESP). This is an open access article under the CC BY-NC-ND license (http://creativecommons.org/licenses/by-nc-nd/4.0/) ,video sharing from mobiles or laptops through the network they need to license by there own cloud system, to update the information and services for the mobile users and suggestion is recomended effectively[5][6][1][10].The first objective of this work is to provide a feature huge for Digital KOT service, to offer efficient management of customers divided into 3, well-known categories as Guest User, Waiters/kitchen, and Account, to build a feature-wealthy for Automatic billing, to offer efficient Kitchen Display of KOT. The second objective of this work is the kitchen display system (KDS) it is digitilized order viewer, it replaces the traditional method of using pen and paper, in addition to it also controls the video of food route system and also display the recipes of the food and its quantity as been display in the system, The kitchen display system machine is incorporated with a cloud-primarily based POS technology because of which it works remotely, and displays orders to be organized as they're fed within the POS software. This reduces both staff effort and the possibilities of manual error with the aid of automating the complete process. The third objective of this work is also combined with online orders like swiggy,zomato to take the order directly from restaurant and delivery order on time without any delay. The fourth objective of this work is to track the daily wastage/extra items been used in the restaurant, a daily stock update is used to control the daily wastage, and also it shows the number of ingredients that are used to prepare the order and also generate the report on daily basis. This software can paintings in any restaurant which has a Wi-Fi facility and tablets having an android operating machine. As the gadget is advanced in android, it becomes smooth for a layman to learn the device. Waiters and executives will find ease in taking orders and speaking with the kitchen, cashiers may be capable of generating correct bills, whereas for admin of eating place it could be easy to view daily logs, reviews, and update databases. Customers might be especially satisfied as there can be no risk of mistakes in their given order, so they'll be assured that the eating place is going to serve them efficiently. Also due to automation in the machine, service can be supplied very quickly.

\section{LITERATURE SURVEY}

In [1]right here utility, a combination of restaurant/hotel management system is provided with internet services.Restaurant/hotel management system combines with the lodge enterprises like the food ordering system, kitchen online ticket(KOT), invoice system and etc., this solution is making a bigger software device in many of restaurant/hotel environment. This device may also rises the quantity and its velocity services. This system may also increase the customer attraction of places in a wide range, deploying this system it may give

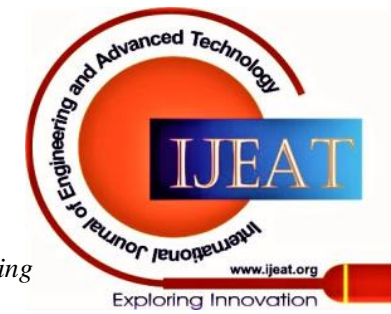




\section{Smart KOT App Generation using an Android Application}

the cost-efficiency possibility and also provide the customer customized services, they will enjoy there needs whenever they need, and also what they need from the restaurant by ordering to settlement and their feedback. In [3] they also carried out work in relation to a restaurant these increase the usage of handheld devices. By using this it overcome from the drawback of old methods like service response time is more during peak hours and also a mismatch of order when there is a rush in the hotel/restaurant. Own cloud is a web application or a software device for what is typically termed, "file hosting". It can keep and serve content from a centralized location, much like Dropbox [6]. The comforted approximately the level of records analysis and surveillance worried in signing up for corporate net services, when the usage of cloud from any business enterprise like Google, Amazon or IBM, there is a prime problem. This means much less protection and privateness for the files which can be stored because the real proprietor of the cloud is stranger or company. To resolve this problem, very own cloud is evolved to save and percentage documents, calendars, and contacts in a stable way, which additionally embraces open standards[8]. Cloud storage offerings [7] are getting increases in most of the cellphones. In a wide area the actual android application, it shows that many of cellphones use the cloud storage it offers the bad usage like it consumes more power usages, the prolonged activity of reaction measures and unnecessary community movement. The main motive of these issues is that current business, where it started develops/build its offerings in many of the cellular plans: on the way it as a speedy with smooth deployment, it completely avoids the customer-aspect system-level report operation for tracking, servicing it is an important part of old method record system, for developing the essential customer-aspect methods in the cellphones designers.

\section{PROBLEM STATEMENT}

Problem Statement that occurs in the current system in the restaurant are such as follow:

- The current ordering system for the restaurant uses the traditional method, for taking order is done manually by the waiters it introduces human errors like order mismatch, billing problem, etc.

- The problems arise to admin/owner during payments because of the order is taken manually by the waiter in a paper.

- During peak hours the service response time is slow because an order is taken manually by a waiter.

- Keeping track of empty, clean, and reserved tables within a restaurant.

- Managers have to analyze hundreds of paper receipts to determine best-selling items, popular hours, and customer satisfaction. They need to re-print the menus when food is not available. This makes it more costly and time-consuming for a restaurant.

\section{PROPOSED SYSTEM}

In the traditional method, the order is taken manually by a waiter through pen and paper, where he generates three bills i.e, one for kitchen purpose, another one for the cashier, and last one for the waiter himself for reference purposes. In the case of the KOT App it consists of three forms namely order, KOT, an order placed . Restaurant KOT generation system is as shown in the Fig. 1.Firstly the order is taken by a waiter thorough tablet, then the order is passed to KOT/Kitchen in the kitchen the other is displayed in a system through KDS. Once the order is complete waiter gets the conformations message in the third step the order conformation is got then food is served to the customer on time without delay and mismatch of orders.

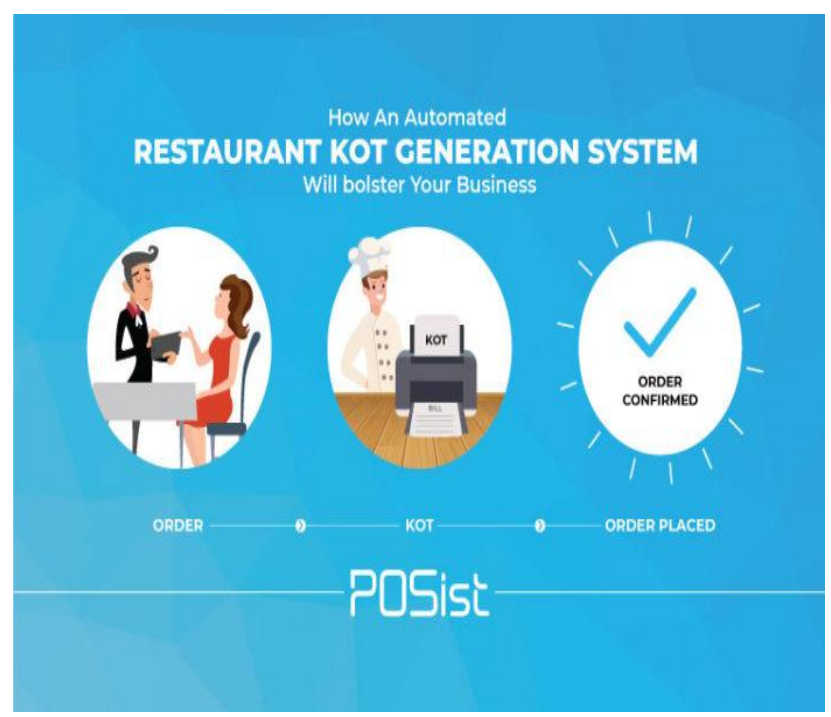

Fig. 1.Restaurant KOT Generation System

The Overview of smart KOT in web application is as shown in Fig.2.The proposed system consists of two forms namely one is android based application and another one is web application. In the case of android based application waiter having an android based tablet where it consists of available food items in a restaurant when the customer arrives at the restaurant, the order is taken by a waiter by using an android tablet.

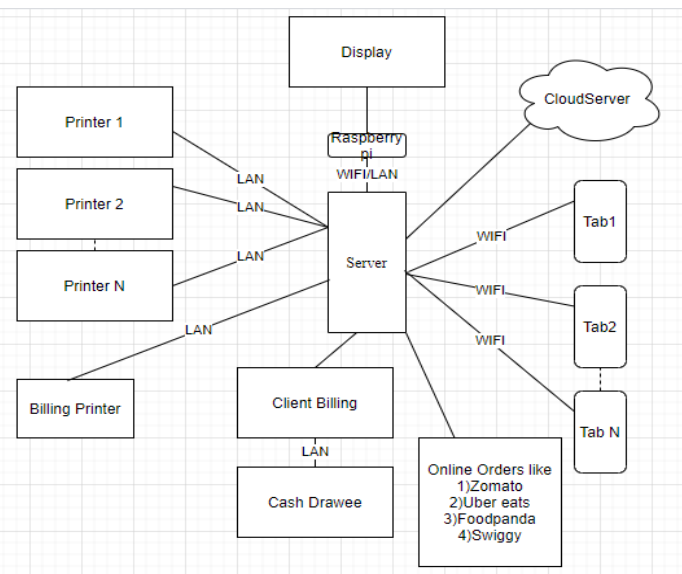

Fig. 2.Overview of Smart KOT in web Application

Web Application is consists of some major features like

- Inventory:

It shows the running order which provides details of the orders currently being made in the restaurant, it also displays the overall orders that are being taken along with the table numbers.

Blue Eyes Intelligence Engineering \& Sciences Publication 
- Kitchen Display System(KDS):

It is a digital order viewer system, whenever the order is placed by the customer it sends to the kitchen. The kitchen contains KDS their order can be view and served on time.

- Online Orders:

Order food online from restaurants, whereas food is delivered, in this project Online order is integrated with a restaurant, online orders like swiggy,zomato, etc.

- Payment Mode:

It includes the details about the payments and expenses made in and/by the hotel. Major features of the proposed system are shown below

\section{- Android Tablet :}

There will be an Android Tablet on every table. This may be used by the customer to check on the food that is available in a restaurant when where they need it. The customer can read the specific menu that they need to order that is generated by the system. The tablet contains the details of the customer and their order details. This helps the admin/owner to generate a proper bill for a particular table without any mismatch of orders and served without delay.

- KOT(Kitchen Online Ticket):

It is noted which is forwarded to the kitchen, cashier/billing system, and one copy remains in the system for the reference purpose. The KOT contains items and their quantity, table number.

\section{- Order Confirmation:}

It is a document that is sent from the waiter to the buyer. It confirms the order has been received and accepted.

\section{IMPLEMENTAION AND RESULTS}

The Proposed system has a web application and an android application in the case of the android app is developed by using the ionic 3 frameworks. Smart KOT app is consists of some of the modules like admin module where it consists of organizations details and, log in details to operates the KOT app once the login details are verified the customer can check on the available table, after selecting the table the customer needs to order the items can be searched by using name and categories, after selecting the item need to placed, placed order is sent to the kitchen after order is sent to multiple kitchens they can view their order in running order module. If in case customers need to cancel the order by using the cancel order model. The web application consists of a configuration model, hotel settings module contains items, category, tables, and kitchen models, payment module where it contains the payment details, and inventory where it contains daily stocks update to the kitchen.

Main modules in the andriod KOT app is shown as follows

\section{- Sync Data:}

Sync data will be used to sync data between kitchenette web application and the android app. If any data changed in the web application user must give sync data in the android app to get it reflected in the app.

- Running Orders:

On clicking running orders, it will go to the running orders page as explained before.

- New Order:

On clicking new order, it will go for taking a new order page i.e. select table page is shown in Fig.4.

Here some of the snapshots of smart KOT app and web application is shown below

\section{Snapshot showing the login page on android application.}

We have to log in with an individual username and password, to keep track of the captains who have placed an order, and we can customize by adding the Client logo. The login page is as shown in Fig.3.

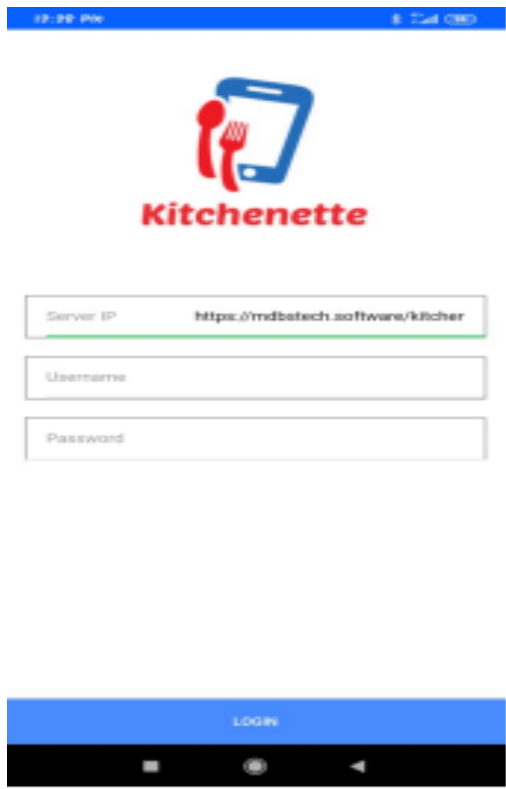

Fig. 3.Login Page

2. Snapshot showing the functionality of "List of a Table" in the android application.

Once login is done, the app will display the tables, the list of tables can be arranged in user compatible. Any number for tables can be added .The list of a table is as shown in Fig.4.

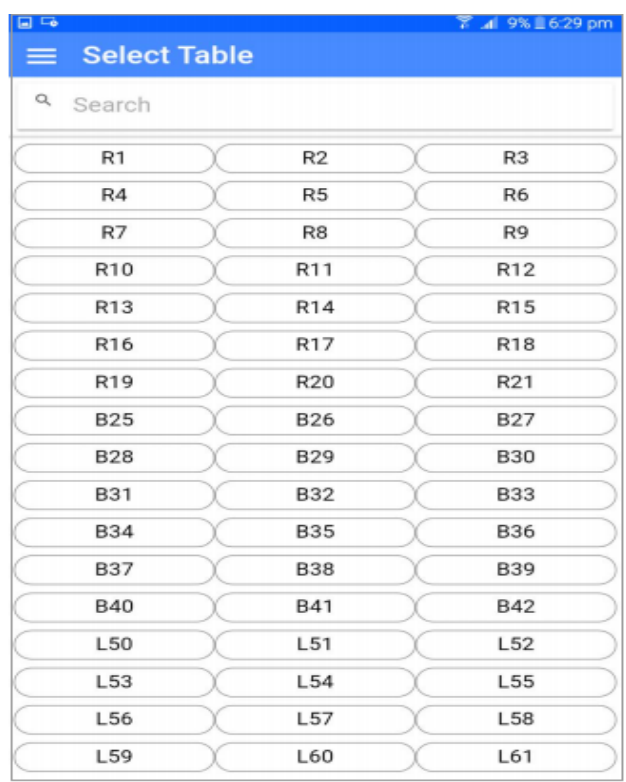

Fig. 4.List of table

3. Snapshot showing the functionality of the "KOT Proceses" in the android application.

This page is an order taking page Once you select the table. Items can be searched using Categories and names. The price of an item also will be visible here for the captains.The KOT process is shown in Fig.5.

Published By: Blue Eyes Intelligence Engineering

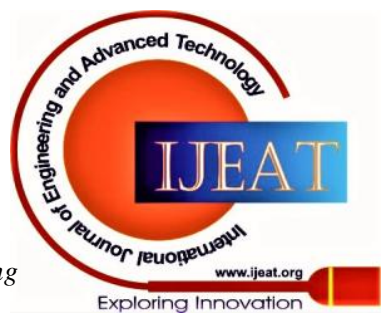




\section{Smart KOT App Generation using an Android Application}

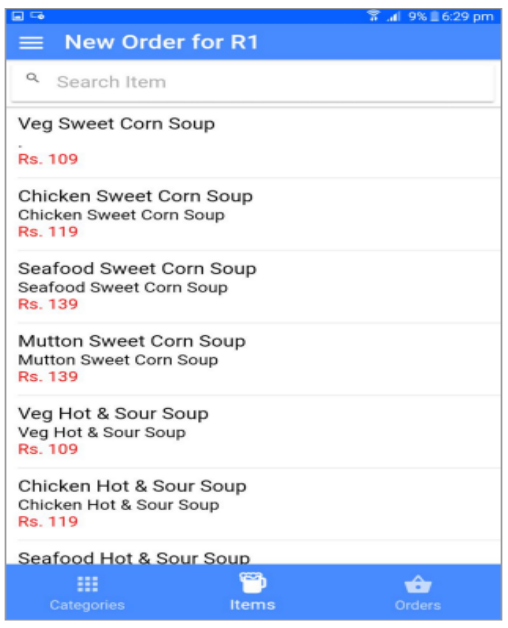

Fig. 5.KOT Proceses

4. Snapshot showing the functionality of "Ordering KOT and Cancel Order" in the android application. We can choose the items just with a click, Items with any category can be selected Click on the button "Send to Kitchen" KOT will be sent to the respective kitchens At one click KOT can be sent to multiple kitchens. The KOT ordering is shown in Fig.6.

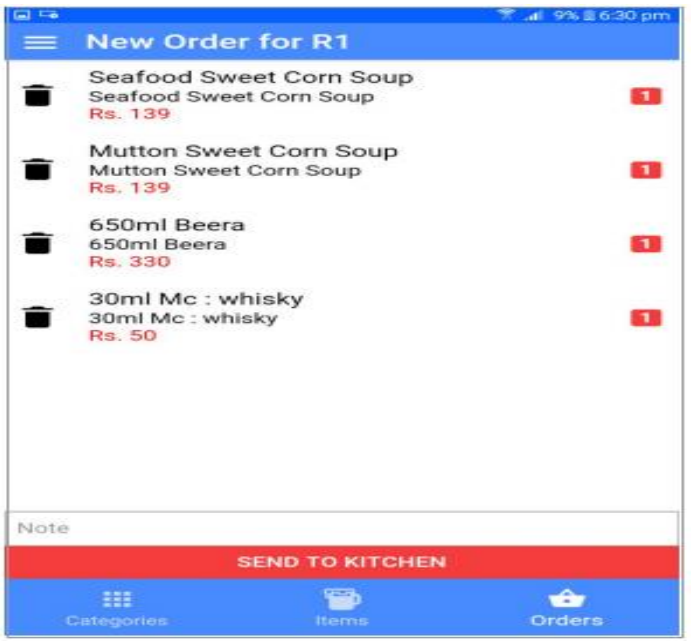

Fig. 6.KOT Ordering

Here we can generate options for cancel KOT by selecting the item and a button will pop with delete option if u click on the delete button, the KOT will be canceled with reason must be mentioned by captains in the bottom to keep track the reason for cancellation.The KOT order cancellation is as shown in Fig.7.

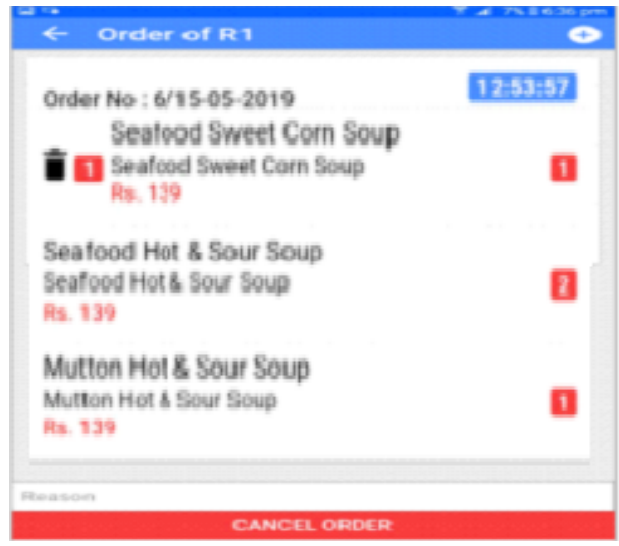

Fig. 7.KOT Order Cancellation

Retrieval Number: F1235089620/2020@BEIESP

DOI: 10.35940/ijeat.F1235.089620

Journal Website: www.ijeat.org
5. Snapshot showing the functionality of "Running orders" in the android application.

By clicking this option you can see the running orders in all the tables Here you can switch to any table to take order by just clicking on the list of tables By clicking on the plus button on the righthand top corner you place the order of the new table.List of running orders is as shown in Fig.8.

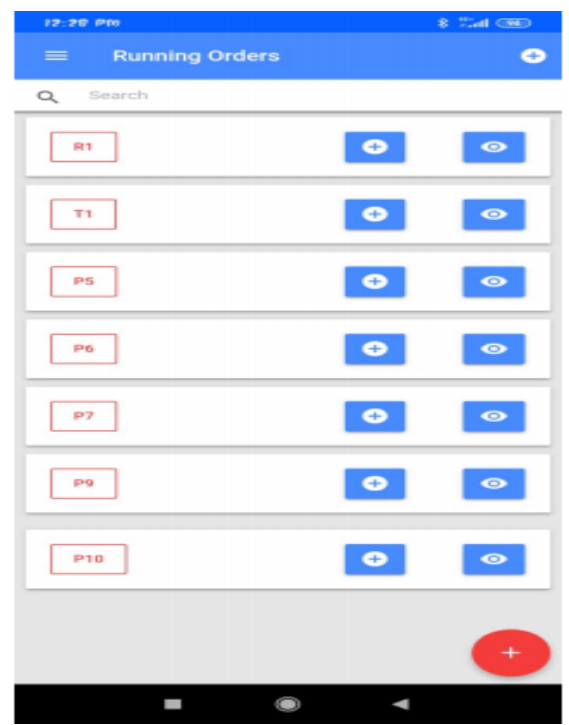

Fig. 8.List of Running Orders

6. Snapshot showing the functionality of "List of orders in the table" in the android application.

If you click on the view button captains can view the previous order placed Captains can also get to the time of the order placed (KOT ordering).List of an order placed in a table is as shown in Fig.9.

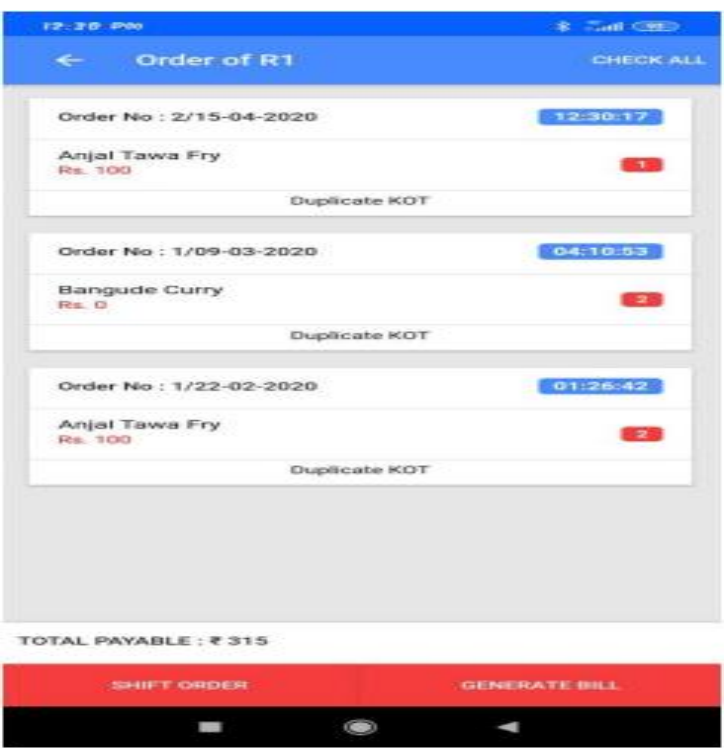

Fig. 9.List of an Order Placed in a Table

7. Snapshot showing the functionality of "Stock update" in KOT app.

It encompasses all of the information about the stock in the hotel. The first option within the Inventory module is the Purchase, which gives information on all the purchases made within the hotel.

Published By:

Blue Eyes Intelligence Engineering \& Sciences Publication

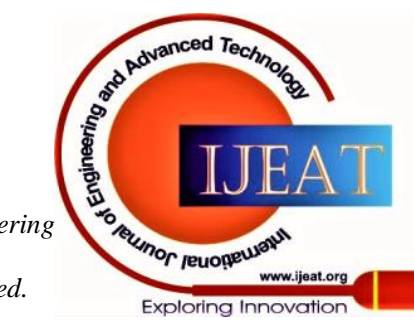


The list displays the overall purchases made in conjunction with the alternatives to edit and print every purchase. The Stock updation is as shown in the Fig.10.

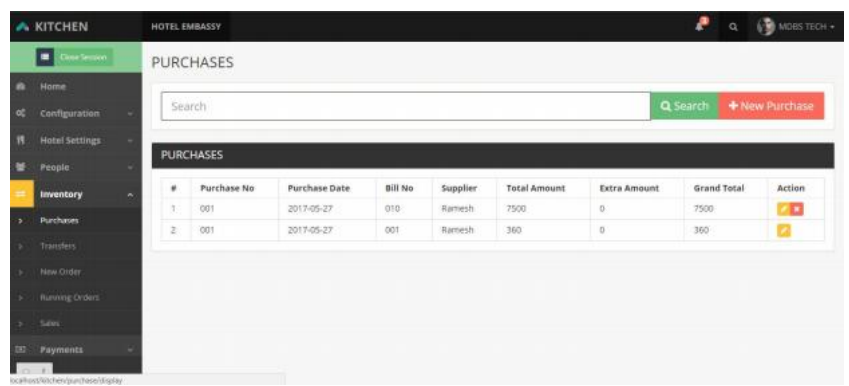

Fig. 10. Stock Updation

\section{CONCLUSION}

The traditional method uses the pen and paper method to overcome from these the smart KOT app is used in this paper, where smart KOT application has categorized into three forms like first is the item that needs to order second whenever the order is placed it sends to KOT/kitchen , third after compilation of order confirmation is message is sent to customers.

\section{FUTURE SCOPE}

In future greater functionalities like lodge management, event management in a restaurant is implemented by the use of a digital smart system that is identically implemented. when the order is completed an alarm is used to send the message of order completion. This work can be implemented by using a wireless alarm. Smart Reserved parking which helps the guests to reserve parking spots in advance of their visit during the peak hours.

\section{ACKNOWLEDGMENT}

My sincere thanks to our Principal, HOD (Department of ECE) of JSS Science and Technological University for the support given to do the project in the company. I would like to thank my guide Mr. Dr M N Jayaram for guidance and support provided. I thank the whole team for the support of technical guidance.

\section{REFERENCES}

1. "Digital Ordering System for Restaurant Using Android" Ashutosh Bhargave, Niranjan Jadhav, Apurva Joshi, Prachi Oke, Prof. Mr. S. R Lahane Department of Computer Engineering, GES's RHSCOE, International Journal of Scientific and Research Publications, Volume 3, Issue 4, April 2013.

2. Kunal Gupta, Stuti Sexena "design and implementation of wireless menu card" 5th International Conference-Confluence The Next Generation Information Technology Summit (Confluence) in 2014.

3. "The Application of Wireless Food Ordering System", Khairunnisa K., Ayob J., Mohd. Helmy A. Wahab, M. Erdi Ayob, M. Izwan Ayob, M. Afif Ayob, MASAUM Journal of Computing, Volume 1 Issue 2, September 2009.

4. Tan-Hsu Tan, Ching-Su Chang, and Yung-Fu Chen,“ Developing an Intelligent e-Restaurant With a Menu Recommender for CustomerCentric Service ", IEEE Transactions on systems, man, and cybernetic, Vol. 42, No. 5, September 2012.

5. Wenjuan Chang, Chunxiao Fan, Junwei Zou, and Xiaoying Zhang, " Design of SCIP System for Push Services Based on Java Card ”, 978-1-4577-0321-8/11, IEEE 2011

6. Http://en.wikipedia.org/wiki/OwnCloud
7. "Improving Cloud Storage Usage Experience for Mobile Applications", Yongshu Bai Xin Zhang Yifan Zhang Binghamton University, Binghamton, New York.

8. "Private Cloud Development to Improve Reliability and Security", Asmaa Qasim Shareef, Ayad A. Abdulsalam, Maha Abd ULatif Sayal, Computer Science Department-University of Baghdad, IRAQ, 2014.

9. N. M. Z. Hashim "Smart Ordering System via Bluetooth" in International Journal of Computer Trends and Technology (IJCTT) volume 4 Issue 7-Month 2013.

10. Annu Lambora, Kunal Gupta "Implementation of Wireless Menu Using IoT",2019 International Conference on Machine Learning, Big Data, Cloud and Parallel Computing (COMITCon).

\section{AUTHORS PROFILE}

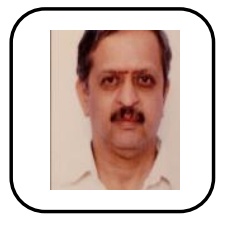

Dr M N Jayaram Assistant Professor at Sri Jaya Chamarajendra College of Engineering Mysuru, Dept of Electronics and Communication. JSS Science and Technology University. He has completed his Bachelor of Engineering in NIE Mysuru and Master of Technology in IISC Bangalore and $\mathrm{PhD}$ in UOM ,Mysuru.

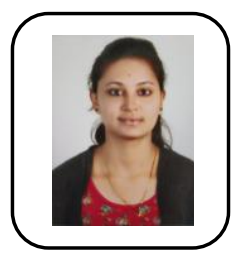

Nisarga Gowda M A MTech student at Sri Jaya Chamarajendra College of Engineering Mysuru in Networking and Internet Engineering, Dept of Electronics and Communication. JSS Science and Technology University. She has completed her Bachelor of Engineering in PES College of Engineering,Mandya.

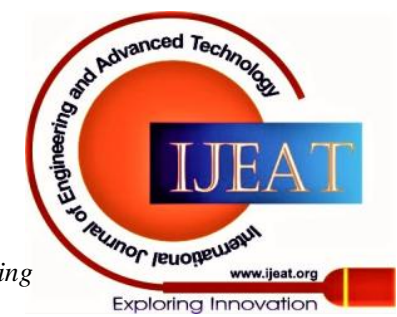

